\title{
Review Article Neuroaging through the Lens of the Resting State Networks
}

\author{
Filippo Cieri $\mathbb{D}^{1}$ and Roberto Esposito $\mathbb{D}^{2}$ \\ ${ }^{1}$ Forensic Mental Health Residential Rehabilitation "Quadrifoglio", Rosello, Chieti, Italy \\ ${ }^{2}$ Department of Radiology, Azienda Ospedaliera Ospedali Riuniti Marche Nord, Pesaro, Italy \\ Correspondence should be addressed to Filippo Cieri; filippocieri@gmail.com
}

Received 1 July 2017; Revised 27 November 2017; Accepted 14 December 2017; Published 15 January 2018

Academic Editor: Hasan Ayaz

Copyright (C) 2018 Filippo Cieri and Roberto Esposito. This is an open access article distributed under the Creative Commons Attribution License, which permits unrestricted use, distribution, and reproduction in any medium, provided the original work is properly cited.

\begin{abstract}
Resting state functional magnetic resonance imaging (rs-fMRI) allows studying spontaneous brain activity in absence of task, recording changes of Blood Oxygenation Level Dependent (BOLD) signal. rs-fMRI enables identification of brain networks also called Resting State Networks (RSNs) including the most studied Default Mode Network (DMN). The simplicity and speed of execution make rs-fMRI applicable in a variety of normal and pathological conditions. Since it does not require any task, rs-fMRI is particularly useful for protocols on patients, children, and elders, increasing participant's compliance and reducing intersubjective variability due to the task performance. rs-fMRI has shown high sensitivity in identification of RSNs modifications in several diseases also in absence of structural modifications. In this narrative review, we provide the state of the art of rs-fMRI studies about physiological and pathological aging processes. First, we introduce the background of resting state; then we review clinical findings provided by rs-fMRI in physiological aging, Mild Cognitive Impairment (MCI), Alzheimer Dementia (AD), and Late Life Depression (LLD). Finally, we suggest future directions in this field of research and its potential clinical applications.
\end{abstract}

\section{Introduction}

Daily life mental activity occurs often in the absence of external stimuli. During this state of consciousness, we are engaged in recording bodily sensations (somesthetic and vegetative); we experience the free association of thoughts that involves memory (past experience, inner dialogue, mental images, and emotions planning future events). In absence of external stimuli, mind jumps from one thought to another with fluidity and simplicity, what William James (1890) called "flow of consciousness." This spontaneous brain activity was called Random Episodic Silent Thinking (REST; [1]), emphasizing the free and errant nature of this mode of thinking, partly in contrast with the engagement of mind during cognitive tasks.

Modern neuroimaging techniques, such as Positron Emission Tomography (PET), Functional Magnetic Resonance Imaging (fMRI), and Magnetoencephalography (MEG), have allowed studying brain in vivo showing the intersection of anatomy and functions. Particularly, fMRI is a relative noninvasive neuroimaging technique used for many years to study brain eloquent areas activation during task execution. In the last two decades fMRI was used to study low-frequency fluctuations of cerebral hemodynamics $(0.01-0.1 \mathrm{~Hz})$ that are associated with complex brain "activation maps" temporally correlated across the brain and correspond to functional RSNs [2]. RSNs are thought to represent the neuronal baseline activity of human brain in the absence of external stimuli and identify the presence of functionally distinct networks $[3,4]$. This brain baseline activity represents a model of mind, enhanced when the focus of attention shifts from external to internal self-referential state [5]. Even if today some skepticism persists about the neural substrate of spontaneous brain activity, this theory is supported not only by rs-fMRI but also by observing electric activity, hemodynamic and metabolic parameters, spontaneous fluctuations of membrane potential, spontaneous spikes, and neurotransmitter release [6]. Despite being the object of a thriving field of clinical research, the investigation of aging through the lens of the RSNs is in its early days. 

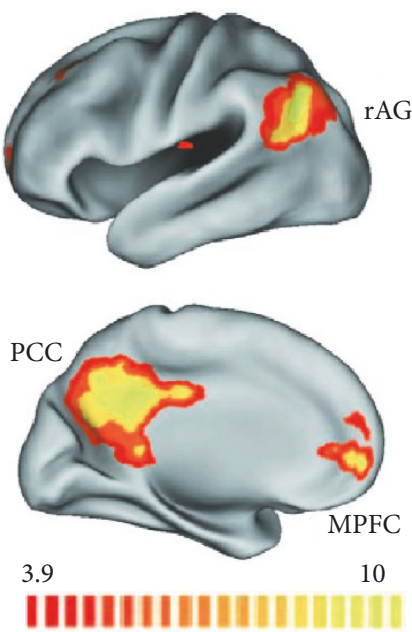

$t$-score

(a)

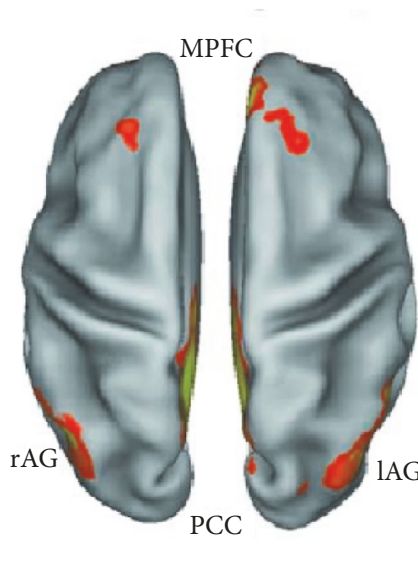

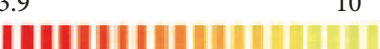

$t$-score

(b)

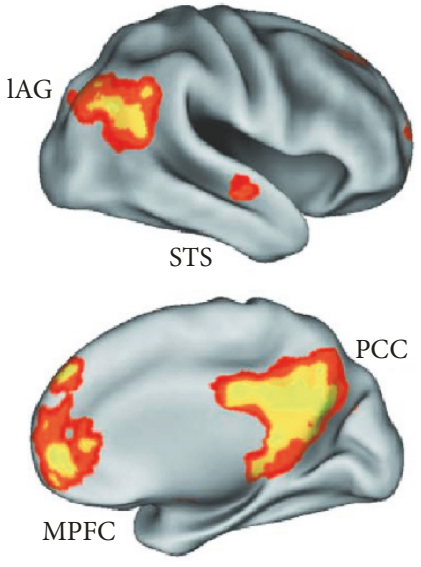

3.9

|

$t$-score

(c)

Figure 1: Default Mode Network (DMN): maps of healthy subjects obtained by means of Independent Component Analysis (ICA) and superimposed on inflated Talairach template: (a) lateral and medial views of the left hemisphere. (b) Dorsal view. (c) Lateral and medial views of the right hemisphere. DMN areas are labeled (MPFC: medial prefrontal cortex; PCC: posterior cingulate cortex; 1AG and rAG: left and right angular gyrus; STS: superior temporal sulcus). The figure is derived from the following study: [7].

\section{Default Mode Network and the Other Resting State Networks}

The identification of the specific brain areas constituting RSNs dates back to early 21st century as a result of a group of scientists using PET imaging $[10,11]$. The first cerebral areas identified constituted a network involving both hemispheres: medial prefrontal cortex (MPFC), the posterior cingulate cortex (PCC), the angular gyrus (AG), and hippocampal regions (HP) (Figure 1). The neural network was called DMN and it is engaged when mental activity is internally directed, for example, when a person is left "undisturbed" to think of himself, about his past or his future. DMN is also referred to as the task-negative network because these regions are typically deactivated during execution of attention demanding tasks $[12,13]$. DMN supports mental processes characterized by future planning, autobiographical memory, moral dilemmas, records of bodily sensations, and self-referential mental activity [14], showing instead reduced activation when a person is engaged in a cognitive task.

Within the DMN the MPFC supports self-referential mental processes, monitoring psychological states [15], and receives interior (bodily sensations, proprioceptive) and external inputs (visual, auditory) supporting their integration and processing. Ventral part of MPFC (VMPFC) shows different interconnections with the limbic system, particularly with amygdala, mediating visceromotor aspects connected to emotions [16], a preconscious process that becomes conscious through modulations of other brain regions. MPFC shows important neural connections with HP; in fact DMN supports memory through two subsystems: the temporalmesial subsystem, associated with mnemonic processes and predominantly made up of HP, that shows high connectivity with PCC/Precuneus (PCC/PrC) and Inferior Parietal Lobule (IPL); the second subsystem connected to the dorsal part of MPFC (DMPFC) is activated during mental situations of self-exploration and sensations. The results suggest that self-referential mental activity engages preferential the MPFC subsystem [17]. Another important DMN region is the Anterior Cingulate Cortex (ACC), associated with the control of different functions such as selection or inhibition of responses, conflict monitoring, and errors detection. Although the precise functions of the DMN remain a matter of debate, mounting evidence points to two distinct DMN subsystems that may mediate dissociable aspects of internal mentation, namely, memory-based construction/simulation (medial temporal lobe subsystem) and introspection about mental states (DMPFC subsystem) $[18,19]$. Stronger resting state functional connectivity within the DMN has been associated with higher frequencies of spontaneous thought and engagement in mind wandering [20]. Nevertheless, regions outside the DMN have also been implicated in mind wandering, in particular executive control systems, yet the exact role of these systems in mind wandering remains not clear [21].

DMN represent only one network, because to date, others RSNs have been identified [3, 22, 23]: the Salience Network (SN); the Frontoparietal Network (lateralized in both hemispheres; FPN); the primary Sensory Motor Network (SMN); the Extrastriate Visual System (EsV); and the Dorsal Attention Network (DAN). RSNs are linked by anatomical connections and engaged in complex patterns of neuronal communication and signaling (Figure 2).

The DAN, which includes Inferior Parietal Sulcus (IPS), Frontal Eye Field (FEF), ACC, and bilateral Middle Temporal Gyrus (MidTempG), has received much attention. It is also 


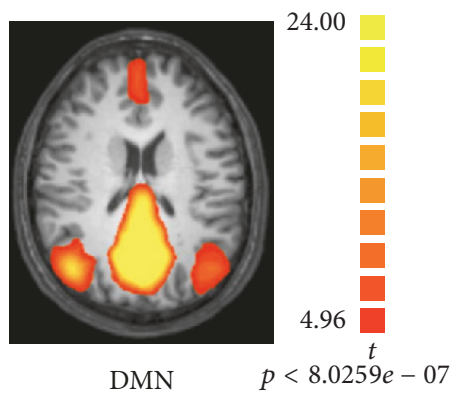

(a)

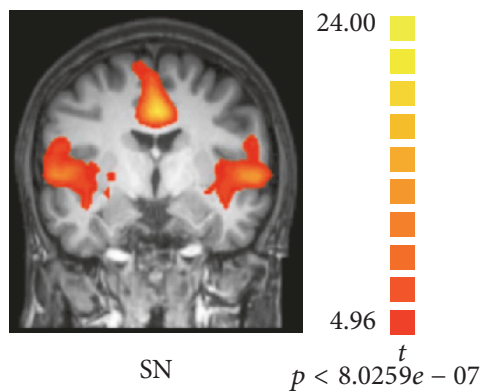

(d)

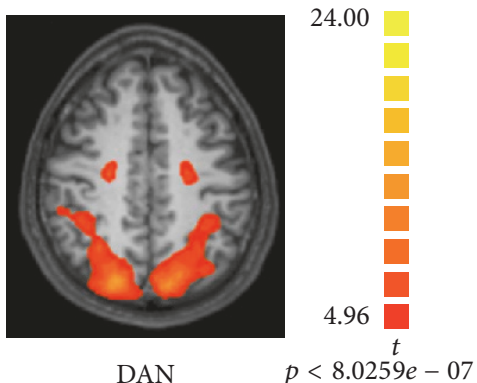

(b)

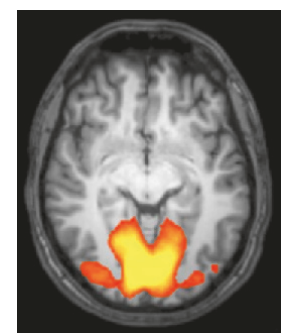

EsV

(e)

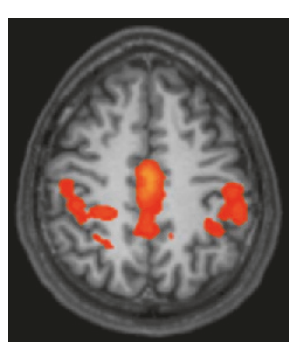

SMN

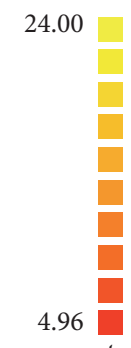

$p<8.0259 e-07$

(c)

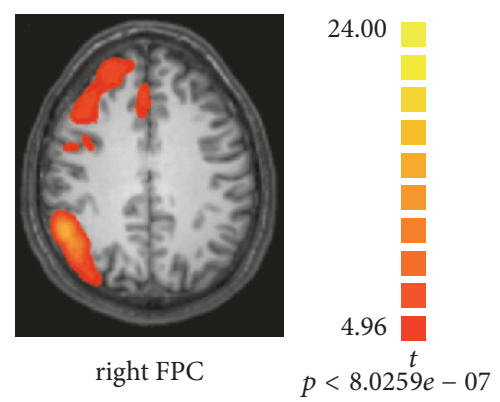

(f)

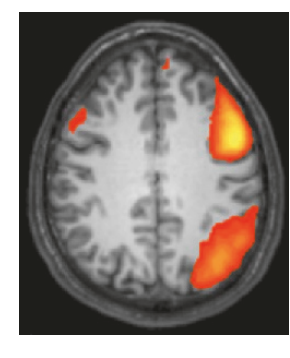

Left FPC
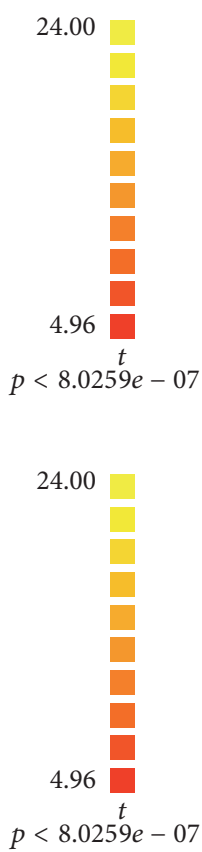

(g)

FIGURE 2: Resting State Networks (RSNs): maps of healthy subjects obtained by means of Independent Component Analysis (ICA), overlaid on Talairach template (T1 weighted images), showed in radiological convention. DMN: Default Mode Network; DAN: Dorsal Attention Network; SMN: Sensory Motor Network; SN: Salience Network; EsV: Extrastriate Visual; FPC: Frontoparietal Control Network. The figure is derived from the following study: [8].

called task-positive network because, as we mentioned above, its main regions are commonly activated in tasks demanding attention and mental control [24, 25]. DMN and DAN show a pattern of anticorrelated activity in both task and resting state studies [26] and their competitive relationship may represent a cerebral mechanism supporting cognitive functions [27, 28], switching focus between internal (supported by DMN) and external channels (supported by DAN) [9], when the system works properly.

Recently, explaining the complex communication between different RSNs has been proposed by a "triple network" hypothesis [29]. This hypothesis involves three networks: central executive network (CEN), SN, and DMN [29, 30]. CEN and SN increase activity during cognitive or affective processes while the DMN shows decreased activation during tasks in which self-referential activity is not involved [31]. Interestingly modifications of interconnections among DMN, CEN, and SN were observed in many psychiatric and neurological disorders, for instance, Attention Deficit Hyperactivity Disorder (ADHD; [32]), psychosis [33], and depression [34], and their functional or dysfunctional activity is crucial during healthy or pathological aging, such as MCI [35] and $\mathrm{AD}$ [36].

\section{Healthy and Pathological Aging}

Many elders, over 65 years old, live in happy and healthy life conditions. Some changes in memory functions are considered a normal part of the aging process, but almost 40 per cent of older adults experience during their life a mild cognitive decline often with an impairment of memory. This impairment may occur in different areas of memory such as visual or verbal memory, visuospatial abilities, immediate memory, or the ability to name objects. When there is no underlying medical condition causing this memory loss, it is known as age-associated memory impairment 
which is considered a part of the normal aging process. These preclinical conditions differ from neurodegenerative diseases like $\mathrm{AD}$ that are characterized by specific biomarkers (neuropsychological, cognitive, biological, biochemical, and neurological).

$\mathrm{AD}$ deeply affects healthy citizen as well as the wealth of public health systems and, with the growing rate of elderly people in western countries, is becoming a health/economic issue of epidemic proportions [38]. A recent intriguing and promising approach in the field of dementia concerns the use of advanced neuroimaging techniques in association with neuropsychological evaluation and quantification of biological markers to identify subjects with high risk of developing $\mathrm{AD}$ in preclinical condition (i.e., Apolipoprotein E4-APOE 4 carriers) $[39,40]$. Indeed, advanced neuroimaging techniques may provide a valid tool to differentiate brain changes due to physiological aging $[41,42]$ and abnormal changes possibly underlying neurodegenerative pathologies such as AD [4345].

Several studies explored modification of DMN connectivity in healthy elders, MCI patients, and $\mathrm{AD}[46,47]$ in order to find useful biomarkers for early diagnosis and start of targeted treatments. DMN functional connectivity impairment correlates with cognitive decline, impairment of executive functions, and working memory [48]. We have strong evidences that allow us to identify specific brain signs prior to the clinical phase of $\mathrm{AD}[9,49]$. In fact, preclinical $\mathrm{AD}$ has been associated with early detection of pathological modifications involving different cerebral regions: reduced DMN connectivity in normal aging and in preclinical stages of dementia [46, 47], especially between anterior and posterior DMN components (MPFC and PCC), was observed. Functional connectivity declines with age mainly affecting DMN; however there are some evidences of increased connectivity with age interpreted as compensatory mechanisms during aging process [50]. Indeed, results observed are controversial, with some studies showing decreased connectivity and other showing opposite results [51], influenced by the data analysis approach used. For example, the seed based method of analysis showed very controversial results with decreased connectivity between HP and PCC or increased connectivity between Prefrontal Cortex (PFC) and HP and between PFC and PCC [52] and the increased connectivity in PFC was interpreted as a compensation mechanism during aging processes. Another interesting approach for rs-fMRI data analysis is the independent component analysis (ICA). The results often show decreased connectivity within DMN, also during tasks, worsening with the onset of $\mathrm{AD}$ [53]. In some studies, the reduction of connectivity of DMN is associated with increased connectivity in other networks such as FPN [54]. The most controversial result is represented by the interpretation of the result observed as prodromal brain changes of neurodegenerative diseases or compensatory mechanisms to counteract cognitive decline $[3,43]$.

As regards $\mathrm{fMRI}$ modifications observed in $\mathrm{AD}$, several studies highlighted the importance of amyloid deposition in specific regions of DMN resulting in decreased functional connectivity. The vulnerability of these regions is unknown; however, it correlated with multiple predisposing factors (vascular damage, iron deposition, and inflammatory changes). The PCC/PrC node of DMN received great attention showing significant modification in normal aging and moreover in $\mathrm{AD}$, indicating amyloid deposition and reduction of metabolism, especially in APOE E4 patients $[49,55]$. PCC/PrC supports self-referential mental activity, memory functions, but also attention, regulating the internal and external focus of thoughts. It has strong functional and anatomical connections with other brain regions, such as the HP, VMPFC, and ACC [55]. The failure of deactivation during task performance [56] and reduction of functional connectivity [57] of PCC may represent an early $\mathrm{AD}$ biomarker. Moreover, PCC is constituted of different functional subparts each one exchanging information with the other RSNs [58]. Rearrangement of DMN intrinsic connectivity especially within PCC and left-IPL may represent a compensatory mechanism to counteract neuronal dysfunction in MCI patients and even more in those MCI patients that during follow-up converted in $\operatorname{AD}[43,47,59]$. As a confounding factor, DMN modifications observed in $\mathrm{AD}$ are common findings in many other mental disorders [60]. Crucially, the extent to which a concurrent task demands attention, and the meta-awareness required to self-identify and self-classify thoughts, restricts its broader application to clinical populations. Evidence suggests that, in the context of low task demands subjects report more off-task thoughts, compared to reports during more demanding tasks [61]. To date, there is a lack of available paradigms to investigate mind wandering in a context free from external additional loads on attention and working memory processes. Also for these reasons mind wandering assessment is particularly difficult in neurodegenerative diseases or psychiatric conditions, where the integrity of the DMN is significantly compromised [60, 62, 63]. Significant intratemporal lobe connectivity, coupled with relatively weaker connectivity of temporal lobe regions to PCC and DMPFC within DMN, is associated with mind wandering [64]. The observed mind wandering frequency is strongly linked to relatively circumscribed connectivity within lateral temporal lobe regions and concomitant decreased connectivity between temporal regions and midline cerebral regions; these results are in contrast with other studies showing increased connectivity within and between a distributed range of DMN nodes during mind wandering $[20,65]$.

An interesting hypothesis that sheds light on aging processes as $\mathrm{AD}$ biomarkers is the modification of DMNDAN anticorrelation pattern [9]. A decreased DMN-DAN anticorrelation seems to be part of the normal aging process and probably its impairment explains partially cognitive decline in MCI and AD patients. In fact, brain is intrinsically organized into anticorrelated networks [26], supporting behaviour and cognitive functions $[27,28]$ and representing a brain physiological function starting up when the focus of attention switches between self-referential mental activity and external attention [66]. Interestingly this DMN-DAN negative correlation modifies its function during life span: it appears during the first year and strengthens during the second year of life [67], becoming more robust in adults to support the development of executive functions and working 
memory [68]. Finally decreased DMN-DAN anticorrelation characterizes healthy elders [69] and may represent neuronal substrates of initial cognitive decline (Figure 3 ).

As concerns the triple network model, altered withinnetwork connectivity among DMN, CEN, and SN was observed in healthy subjects at risk of AD (APOE4 carriers) [33]. The preserved connectivity within triple network system may explain preserved cognition in the cognitively normal APOE4 allele carriers. Moreover, the normal functioning of triple network interaction may positively affect the compensation of initial cognitive decline as observed in a recent study showing reduced connectivity within DMN between PCC and HP and from thalamus to PCC and increased connectivity within SN from dorsal ACC to striatum, from the CEN to the DMN, and from the SN to CEN in MCI patients compared with AD patients and healthy controls [67].

Finally, interesting results arise from the intersection of imaging data and molecular data in normal aging and $\mathrm{AD}$ tracing by means of PET amyloid a $\beta 1-42$ (Pittsburgh Compound $\mathrm{B}(\mathrm{PiB})-\mathrm{PET})$ and Tau neurofibrillary tangles (AV1451-PET). Indeed, individuals with preclinical AD have relied on associations with in vivo measures of amyloid pathology. While many studies have reported decreased functional connectivity with increased amyloid $(\mathrm{a} \beta)$ burden in the medial temporal lobe, posterior midline, and parietal regions [38], other studies have reported regions of both increased and decreased connectivity with elevated amyloid [70]. With the recent advent of in vivo Tau-PET tracers it is now possible to extend investigations on fcMRI in a sample of cognitively normal elderly humans to regional measures of Tau. In a recent study [71], the authors showed that amyloidpositive $(\mathrm{a} \beta+)$ individuals were characterized by increased connectivity in DMN and SN when neocortical Tau levels are low, whereas a $\beta+$ individuals demonstrate decreased connectivity in these networks as a function of elevated Tau-PET signal. This pattern suggests a hyperconnectivity phase followed by a hypoconnectivity phase in the course of preclinical AD [71].

\section{Late Life Depression}

Another important condition that affects people after 60-65 years of age, with emotional and cognitive impairment, is Late Life Depression (LLD), a common mental disability in elderly population characterized by the presence of depressed mood, loss of interest or pleasure in daily activities, sleep disturbance, appetite disturbance, and cognitive and somatic symptoms. This specific mood disorder tends to increase considering the progressive aging population. Its prevalence rates can range from $1 \%$ to $4 \%$ for major and up to $13 \%$ for minor depression [72]. FMRI studies have shown varied and controversial results reporting modifications of cerebral connectivity in elders with depression $[73,74]$, but there is a strong association between depressive symptoms and MCI among older adults, although the neural correlates of this relationship are not fully understood. We surely agree that patients with LLD show often signs of cognitive decline and patients with MCI can complain about anxiety or depression symptoms; most importantly, both show higher conversion rate to $\mathrm{AD}[75]$.

One of most important signs of these patients is the lack of DMN suppression during cognitive tasks [76], closely linked to the enhanced ruminative processes. Indeed, these patients demonstrate more engagement in mentalizing about the self and past autobiographical experiences, focusing on negative thoughts. The DMN area most involved in depressed patients is the MPFC, including the ventromedial and dorsolateral part. As we have mentioned above MPFC supports selfreferential mental processes, monitoring psychological states [15]; it is critical to social cognition, self-reference, emotional decision-making, and emotion regulation. This region has been implicated not only in LLD, but more in general in the development of anxiety and depression disorders [77, 78]. The functions of this area are tied to its structural and functional connections to numerous key areas of DMN such as the PCC/PrC and HP, key nodes of memory retrieval, as well as the amygdala and insula, key region of the $\mathrm{SN}$, caudate and putamen, core of the reward system, superior temporal sulcus (STS) and middle temporal cortex, and central nodes of theory of mind [79]. The involvement of all these structures and functions explains the extended presence of such complex and disabling affective, cognitive, and somatic symptomatology. It is not fully understood whether the DMPFC is more an "affective" region and the VMPFC is more an "emotion regulation" region or vice versa, but Aghajani and colleagues [80], for example, propose the DMPFC as an emotional assessment/processing region and the VMPFC with functions of emotions regulation. The increased connectivity shown by these patients between specific nodes of DMN, as the subgenual part of ACC (sgACC) and the PCC, could be detrimental to cognitive processes $[60,81]$. However, connectivity between the SN (i.e., anterior insula, dorsal ACC) and the anterior nodes of DMN [82] as well as between the insula and the amygdala [83] seems enhanced in depressed individuals when compared with controls and these results are coherent with the more engagement in mentalizing about the self and past autobiographical experiences and focusing on negative thoughts demonstrated in these patients. In our recent study [37] we showed a significant modification in the left FPN (IFPN) characterized by increased connectivity in the left Superior Parietal Lobule (SPL) and left DLPFC in LLD group (Figure 4). The IFPN is implicated in language and working memory processing $[84,85]$ and the significant modification found in our study could justify some typical cognitive symptoms in LLD, assuming an inability to regulate the activation of an important area for specific cognitive tasks, as the case of working memory, impaired in LLD, MCI, and AD. Alterations in SPL connectivity are less often described in the literature to be associated with LLD [86], while the structural connectivity using Diffusion Tensor Imaging (DTI) between SPL of both cerebral hemispheres seems to discriminate patients with depression from healthy controls [87]. Parietal cortex provides a coherent self-representation across space and time [88] and is implicated in top-down control of attention, with sensorimotor integration, balancing internally and externally directed attention [58]. In a recent 


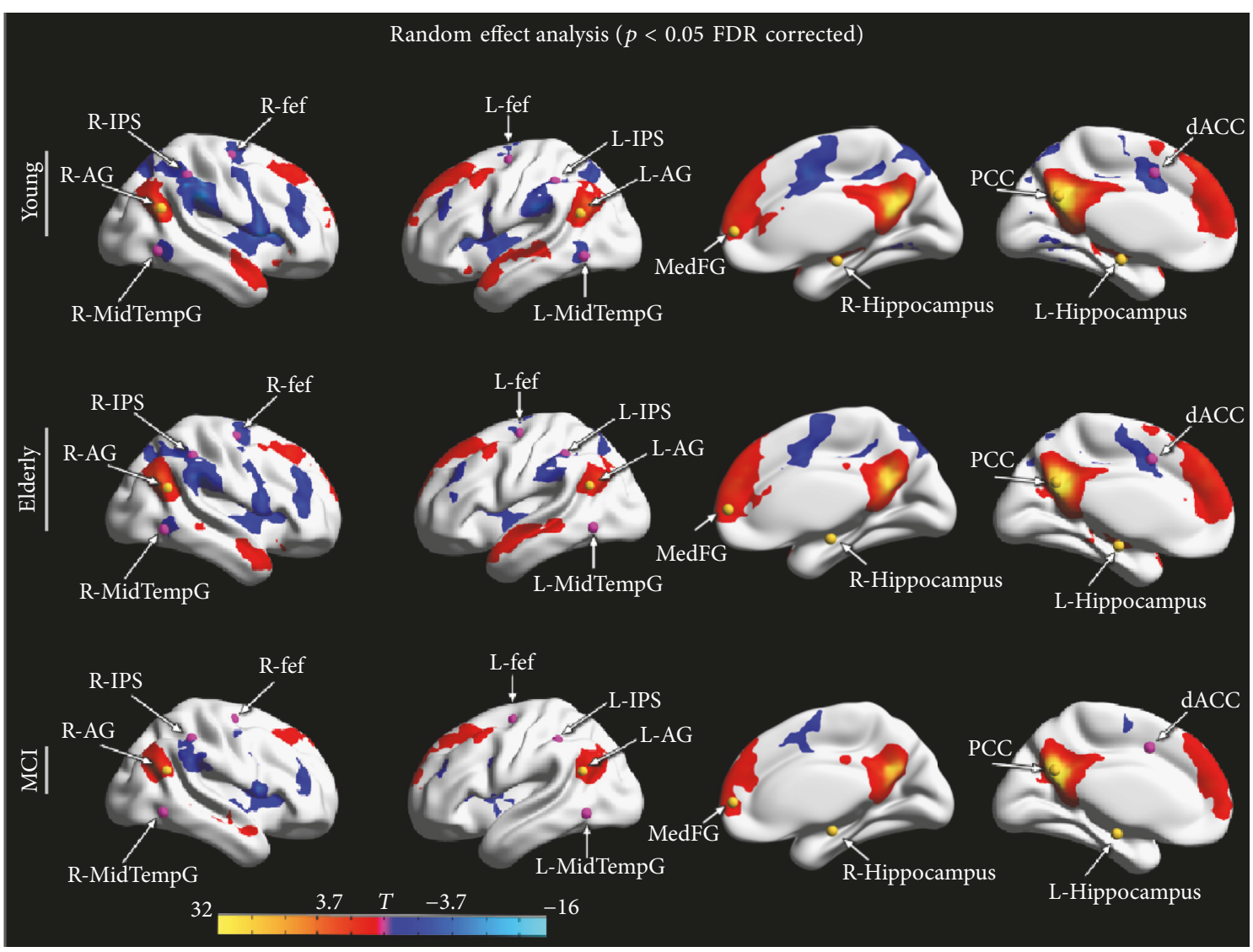

Figure 3: Default Mode Network (DMN) and Dorsal Attention Network (DAN) anticorrelations: Seed based connectivity maps obtained from random effects group analyses, superimposed on inflated Talairach template. The maps show DMN-DAN anticorrelations for healthy young subjects, healthy elders, and Mild Cognitive Impairment (MCI) patients. The figure is derived from the following study: [9].

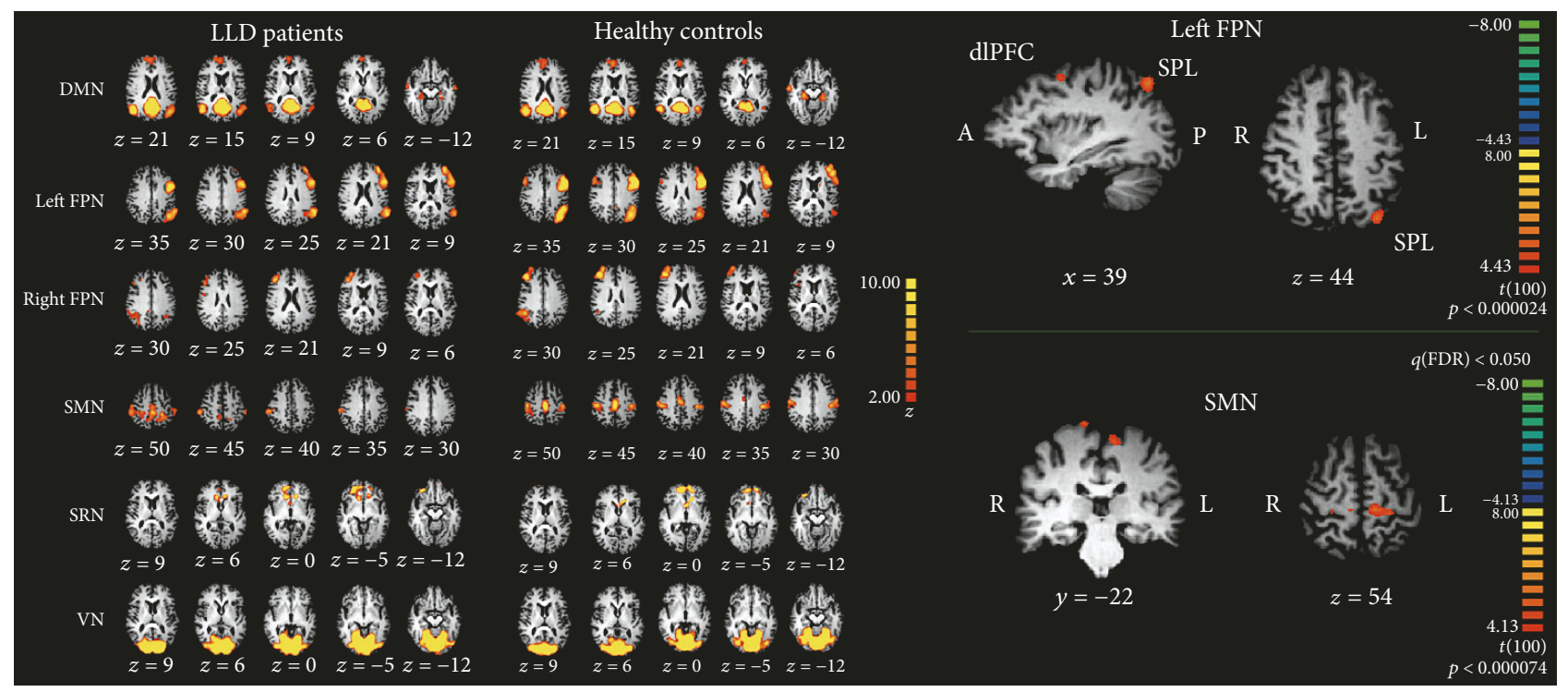

Figure 4: Resting State Networks (RSNs) in Late Life Depression (LLD): left panel illustrates Default Mode Network (DMN), Somatomotor Network (SMN), left and right Frontoparietal Network (FPN), Self-Referential Network (SRN), and Visual Network (VN), overlaid on Talairach template (T1 weighted images), showed in radiological convention. Left: LLD patients; Right: healthy controls. Right panel illustrates between-group differences. Top: Left FPN; Down: SMN. Contrast maps are overimposed on Talairach template (T1 weighted images) in radiological convention. The figures are derived from the following study: [37]. 
comparative study between schizophrenic patients and major depressive disorder, the authors found significant reduction in connectivity within the posterior nodes of DMN and SPL in patients with depression hypothesizing an imbalance between internally and externally directed attention and mental state attribution [89]. In fact, SPL appears to be involved in disengaging or maintaining attention to visual and tactile stimuli [90, 91], as demonstrated in patients with tumor localized in left SPL, suggesting that SPL is important in maintaining internal representation of body states [92]. This region continues onto the medial surface of the hemisphere as the PrC, forming rectangular-shaped area involved in mental imagery and recall of personal experiences; thus, like the MPFC, it is part of the DMN of the brain, engaged during activities such as daydreaming and introspection [93], both impaired in depression disorders. Also, epileptic seizures in the SPL cause disturbances of the body image; thus it is also possible to hypothesize an increased attention for the internal representation of body states in patients with LLD.

\section{Conclusion}

For many years, modern neuroimaging techniques neglected brain spontaneous activity, focusing only on changes evoked by external tasks. Within the past two decades, rs-fMRI has taken off as a major tool to study brain in vivo, especially for those patients less cooperative, offering detailed and clear information about the spontaneous brain dynamics in both physiological and pathological conditions. Indeed, the common target of the neuroscientists is to identify early biomarkers before clinical outbreak of $\mathrm{AD}$ and advancements in genetics, neurobiology, and neuroimaging techniques allowed researchers to hypothesize the mechanisms underlying these disorders.

Abnormal functional connectivity has been reported in several neurologic diseases even if these results remain controversial. In the first rs-fMRI studies the results showed intranetwork connectivity modifications while more recent studies underlie altered interactions between different RSNs. In recent years, neurological disorders have better understood in its specific neural correlates and some shared results have been achieved, as, for example, the reduced DMN connectivity in normal aging and in preclinical stages of dementia $[46,47]$, especially between anterior and posterior DMN components. Moreover, the posterior nodes of DMN have received great attention showing significant modification in normal aging and moreover in $\mathrm{AD}$, indicating amyloid deposition and reduction of metabolism, also in preclinical conditions as observed in APOE E4 patients [49, 51]. Other investigations in this area have demonstrated that $\mathrm{PCC} / \mathrm{PrC}$ supports self-referential mental activity, memory functions, but also attention in regulation of internal and external focus of thoughts. Thus, the lack of this deactivation during task performance [56] and the reduction of functional connectivity [57] of PCC may represent an early biomarker of AD.

Another important chapter within the understanding of healthy and pathological aging has been the anticorrelation of task-negative and task-positive networks. In fact, decreased
DMN-DAN anticorrelation has been observed in a recent study comparing young subjects, healthy elders, and MCI patients [69] hypothesizing that reduced anticorrelations may be considered a neuronal substrate of aging brain supporting cognitive decline. As concerns the triple network model, healthy subjects at risk of AD (APOE4 carriers) have shown altered within-network connectivity among DMN, CEN and SN [33]. The preserved connectivity within triple network system may explain preserved cognition in the cognitively normal APOE4 individuals.

Finally, another disease recently investigated through the rs-fMRI in aging is LLD, with important emotional and cognitive impairment. One of the most important areas involved in the LLD is the MPFC, for its implication in typical mental activity in depressed patients, such as selfreference, social cognition, emotional decision-making, and emotion regulation. LLD patients are more engaged in metalizing about self and past autobiographical experiences and focusing on negative thoughts. These individuals are characterized by inability to suppress DMN activity during cognitive task, coherent with these enhanced overthinking processes. Connectivity between SN and the anterior nodes of DMN as well as between the insula and the amygdala is a second feature coherent with their tendency to ruminate. Moreover, reduction in connectivity within the posterior nodes of DMN and SPL was observed in depressed patients, coherent with an imbalance between internally and externally directed attention and mental state attribution [89]. This imbalance seems one of the causes of cognitive impairment as a consequence of inability to focus on a specific cognitive task, because the attentive resources are disengaged and hijacked on emotional aspects, driving the ruminative elaborations and this is crucial and detrimental to cognitive processes.

Even if rs-fMRI represents a promising tool to study brain there are many confounding factors to be clarified [94]. For example, there is no control system to check if patients fall asleep during MRI scans; moreover, the great variety of methods of MRI data analysis contributes to create an "excess" of heterogeneity of results. Finally, fMRI records brain modifications of neurovascular coupling BOLD signal. Several diseases modify this signal with a consequent nonquantifiable effect on BOLD signal. Future research will take benefit by the combination of different methods such as MEG, Electroencephalography (EEG) and cerebral perfusion, and molecular data.

Functional connectivity has contributed substantially to the field of Alzheimer's research showing cerebral modifications even before symptoms arise and is emerging as a promising biomarker for longitudinal studies.

\section{Conflicts of Interest}

The authors declare that there are no conflicts of interest regarding the publication of this paper.

\section{Authors' Contributions}

Filippo Cieri and Roberto Esposito equally contributed to this work. 


\section{References}

[1] N. C. Andreasen, D. S. O’Leary, T. Cizadlo et al., "Remembering the past: Two facets of episodic memory explored with positron emission tomography," The American Journal of Psychiatry, vol. 152, no. 11, pp. 1576-1585, 1995.

[2] J. S. Damoiseaux, S. A. R. B. Rombouts, F. Barkhof et al., "Consistent resting-state networks across healthy subjects," Proceedings of the National Acadamy of Sciences of the United States of America, vol. 103, no. 37, pp. 13848-13853, 2006.

[3] G. Deco, V. K. Jirsa, and A. R. McIntosh, "Emerging concepts for the dynamical organization of resting-state activity in the brain," Nature Reviews Neuroscience, vol. 12, no. 1, pp. 43-56, 2011.

[4] S. M. Smith, P. T. Fox, K. L. Miller et al., "Correspondence of the brain's functional architecture during activation and rest," in Proceedings of the National Academy of Sciences of the United States of America, vol. 106, pp. 13040-13045, 2009.

[5] S. Arslan, S. I. Ktena, A. Makropoulos, E. C. Robinson, D. Rueckert, and S. Parisot, "Human brain mapping: A systematic comparison of parcellation methods for the human cerebral cortex," in Neuroimage, A systematic comparison of parcellation methods for the human cerebral cortex. Neuroimage, 2017.

[6] C. O'Donnell and M. C. W. van Rossum, "Systematic analysis of the contributions of stochastic voltage gated channels to neuronal noise," Frontiers in Computational Neuroscience, vol. 8, no. 105, 2014.

[7] R. Esposito, P. A. Mattei, C. Briganti et al., "Modifications of default-mode network connectivity in patients with cerebral glioma," PLoS ONE, vol. 7, no. 7, Article ID e40231, 2012.

[8] R. Esposito, F. Cilli, V. Pieramico et al., "Acute effects of modafinil on brain resting state networks in young healthy subjects," PLoS ONE, vol. 8, no. 7, Article ID e69224, 2013.

[9] R. Esposito, F. Cieri, P. Chiacchiaretta et al., "Modifications in resting state functional anticorrelation between default mode network and dorsal attention network: comparison among young adults, healthy elders and mild cognitive impairment patients," Brain Imaging and Behavior, pp. 1-15, 2017.

[10] G. L. Shulman, J. A. Fiez, M. Corbetta et al., "Common blood flow changes across visual tasks: II. Decreases in cerebral cortex," Journal of Cognitive Neuroscience, vol. 9, no. 5, pp. 648663, 1997.

[11] M. E. Raichle, A. M. MacLeod, A. Z. Snyder, W. J. Powers, D. A. Gusnard, and G. L. Shulman, "A default mode of brain function," Proceedings of the National Acadamy of Sciences of the United States of America, vol. 98, no. 2, pp. 676-682, 2001.

[12] K. Mevel, B. Landeau, M. Fouquet et al., "Age effect on the default mode network, inner thoughts, and cognitive abilities," Neurobiology of Aging, vol. 34, no. 4, pp. 1292-1301, 2013.

[13] S. Passow, K. Specht, T. C. Adamsen et al., "Default-mode network functional connectivity is closely related to metabolic activity," Human Brain Mapping, vol. 36, no. 6, pp. 2027-2038, 2015.

[14] R. L. Buckner, "The brain's default network: origins and implications for the study of psychosis," Dialogues in Clinical Neuroscience, vol. 15, no. 3, pp. 351-358, 2013.

[15] K. L. Phan, T. Wager, S. F. Taylor, and I. Liberzon, "Functional neuroanatomy of emotion: a meta-analysis of emotion activation studies in PET and fMRI," NeuroImage, vol. 16, no. 2, pp. 331-348, 2002.
[16] G. Northoff, "Is the self a higher-order or fundamental function of the brain? The "basis model of self-specificity" and its encoding by the brain's spontaneous activity," Cognitive Neuroscience, vol. 7, no. 1-4, pp. 203-222, 2016.

[17] K. K. Szpunar, J. M. Watson, and K. B. McDermott, "Neural substrates of envisioning the future," Proceedings of the National Acadamy of Sciences of the United States of America, vol. 104, no. 2, pp. 642-647, 2007.

[18] J. R. Andrews-Hanna, J. Smallwood, and R. N. Spreng, "The default network and self-generated thought: component processes, dynamic control, and clinical relevance," Annals of the New York Academy of Sciences, vol. 1316, no. 1, pp. 29-52, 2014.

[19] R. L. Buckner, “The serendipitous discovery of the brain's default network," NeuroImage, vol. 62, no. 2, pp. 1137-1145, 2012.

[20] L. Yang, F. Lin, Y. Zhou et al., "Iterative cross-correlation analysis of resting state functional magnetic resonance imaging data," PLoS ONE, vol. 8, no. 3, p. e58653, 2013.

[21] K. C. R. Fox, R. N. Spreng, M. Ellamil, J. R. AndrewsHanna, and K. Christoff, "The wandering brain: meta-analysis of functional neuroimaging studies of mind-wandering and related spontaneous thought processes," Neuroimage, vol. 111, pp. 611-621, 2015.

[22] M. P. van den Heuvel and H. E. Hulshoff Pol, "Exploring the brain network: a review on resting-state fMRI functional connectivity," European Neuropsychopharmacology, vol. 20, no. 8, pp. 519-534, 2010.

[23] A. Mitra and M. E. Raichle, "How networks communicate: propagation patterns in spontaneous brain activity," Philosophical Transactions of the Royal Society of London. Series B, Biological Sciences, vol. 371, no. 1705, 2016.

[24] M. Corbetta and G. L. Shulman, "Control of goal-directed and stimulus-driven attention in the brain," Nature Reviews Neuroscience, vol. 3, no. 3, pp. 201-215, 2002.

[25] F. X. Castellanos, S. Cortese, and E. Proal, "Connectivity," in Current Topics in Behavioral Neurosciences, vol. 16, pp. 49-77, 2014.

[26] P. Fransson, "Spontaneous low-frequency BOLD signal fluctuations: an fMRI investigation of the resting-state default mode of brain function hypothesis," Human Brain Mapping, vol. 26, no. 1, pp. 15-29, 2005.

[27] L. Q. Uddin, A. M. C. Kelly, B. B. Biswal, F. X. Castellanos, and M. P. Milham, "Functional connectivity of default mode network components: correlation, anticorrelation, and causality," Human Brain Mapping, vol. 30, no. 2, pp. 625-637, 2009.

[28] K. Gopinath, V. Krishnamurthy, R. Cabanban, and B. A. Crosson, "Hubs of anticorrelation in high-resolution restingstate functional connectivity network architecture," Brain Connectivity, vol. 5, no. 5, pp. 267-275, 2015.

[29] V. Menon, "Large-scale brain networks and psychopathology: a unifying triple network model," Trends in Cognitive Sciences, vol. 15, no. 10, pp. 483-506, 2011.

[30] V. Menon and L. Q. Uddin, "Saliency, switching, attention and control: a network model of insula function," Brain Structure and Function, vol. 214, no. 5-6, pp. 655-667, 2010.

[31] M. D. Greicius and V. Menon, "Default-mode activity during a passive sensory task: uncoupled from deactivation but impacting activation," Cognitive Neuroscience, vol. 16, no. 9, pp. 14841492, 2004.

[32] Y. F. Zang, Y. He, C. Z. Zhu et al., "Altered baseline brain activity in children with ADHD revealed by resting-state functional MRI," Brain \& Development, vol. 29, pp. 83-91, 2007. 
[33] S. Khadka, S. A. Meda, M. C. Stevens et al., "Is aberrant functional connectivity a psychosis endophenotype? A resting state functional magnetic resonance imaging study," Biological Psychiatry, vol. 74, no. 6, pp. 458-466, 2013.

[34] H. Zheng, L. Xu, F. Xie et al., "The Altered Triple Networks Interaction in Depression under Resting State Based on Graph Theory," BioMed Research International, vol. 2015, Article ID 386326, 8 pages, 2015.

[35] S. Cai, T. Chong, Y. Peng et al., "Altered functional brain networks in amnestic mild cognitive impairment: a resting-state fMRI study," Brain Imaging and Behavior, vol. 11, no. 3, pp. 619631, 2017.

[36] X. Wu, Q. Li, X. Yu et al., "A triple network connectivity study of large-scale brain systems in cognitively normal APOE4 carriers," Frontiers in Aging Neuroscience, vol. 8, article no. 231, 2016.

[37] F. Cieri, R. Esposito, N. Cera, V. Pieramico, A. Tartaro, and M. di Giannantonio, "Late-Life Depression: Modifications of Brain Resting State Activity," Journal of Geriatric Psychiatry and Neurology, vol. 30, no. 3, pp. 140-150, 2017.

[38] M. D. Hurd, P. Martorell, A. Delavande, K. J. Mullen, and K. M. Langa, "Monetary costs of dementia in the United States," The New England Journal of Medicine, vol. 368, no. 14, pp. 1326-1334, 2013.

[39] D. M. Holtzman, E. Mandelkow, and D. J. Selkoe, "Alzheimer disease in 2020," Cold Spring Harbor Perspectives in Medicine, vol. 2, no. 11, 2012.

[40] J. Kim and W. Pan, "Highly adaptive tests for group differences in brain functional connectivity," NeuroImage: Clinical, vol. 9, pp. 625-639, 2015.

[41] M. R. Brier, J. B. Thomas, A. M. Fagan et al., "Functional connectivity and graph theory in preclinical Alzheimer's disease," Neurobiology of Aging, vol. 35, no. 4, pp. 757-768, 2014.

[42] R. F. Buckley, A. P. Schultz, T. Hedden et al., "Functional network integrity presages cognitive decline in preclinical Alzheimer disease," Neurology, vol. 89, no. 1, pp. 29-37, 2017.

[43] R. Esposito, A. Mosca, V. Pieramico, F. Cieri, N. Cera, and S. L. Sensi, "Characterization of resting state activity in MCI individuals," PeerJ, vol. 2013, no. 1, article no. e135, 2013.

[44] K. N. Dillen, H. I. Jacobs, J. Kukolja et al., "Functional disintegration of the default mode network in Prodromal Alzheimer's Disease," Journal of Alzheimer's Disease, vol. 59, no. 1, pp. 169187, 2017.

[45] S. Kazemifar, K. Y. Manning, N. Rajakumar et al., "Spontaneous low frequency BOLD signal variations from resting-state fMRI are decreased in Alzheimer disease," PLoS ONE, vol. 12, no. 6, Article ID e0178529, 2017.

[46] Y. Ouchi and M. Kikuchi, "A review of the default mode network in aging and dementia based on molecular imaging," Reviews in the Neurosciences, vol. 23, no. 3, pp. 263-268, 2012.

[47] X. Song, A. Mitnitski, N. Zhang, W. Chen, and K. Rockwood, "Dynamics of brain structure and cognitive function in the Alzheimer's disease neuroimaging initiative," Journal of Neurology, Neurosurgery \& Psychiatry, vol. 84, no. 1, pp. 71-78, 2013.

[48] D. Vidal-Pineiro, C. Valls-Pedret, and S. Fernandez-Cabello, "Decreased default mode network connectivity correlates with age-associated structural and cognitive changes," Frontiers in Aging Neuroscience, vol. 6, article no. 256, 2014.

[49] R. A. Sperling, P. S. LaViolette, K. O'Keefe et al., "Amyloid deposition is associated with impaired default network function in older persons without dementia," Neuron, vol. 63, no. 2, pp. 178-188, 2009.
[50] E. L. Dennis and P. M. Thompson, "Functional brain connectivity using fMRI in aging and Alzheimer's disease," Neuropsychology Review, vol. 24, no. 1, pp. 49-62, 2014.

[51] N. Filippini, L. D. Nickerson, C. F. Beckmann et al., "Age-related adaptations of brain function during a memory task are also present at rest," NeuroImage, vol. 59, no. 4, pp. 3821-3828, 2012.

[52] J. P. F. Bai, R. Bell, S. Buckman et al., "Translational biomarkers: From preclinical to clinical a report of 2009 AAPS/ACCP biomarker workshop," The AAPS Journal, vol. 13, no. 2, pp. 274283, 2011.

[53] M. Demirtas, C. Falcon, A. Tucholka, J. Gispert, J. L. Molinuevo, and G. Deco, "A whole-brain computational modeling approach to explain the alterations in resting-state functional connectivity during progression of Alzheimer's disease," NeuroImage: Clinical, vol. 16, pp. 343-354, 2017.

[54] F. Agosta, M. Pievani, C. Geroldi, M. Copetti, G. B. Frisoni, and M. Filippi, "Resting state fMRI in Alzheimer's disease: beyond the default mode network," Neurobiology of Aging, vol. 33, no. 8, pp. 1564-1578, 2012.

[55] F. De Vogelaere, P. Santens, E. Achten, P. Boon, and G. Vingerhoets, "Altered default-mode network activation in mild cognitive impairment compared with healthy aging," Neuroradiology, vol. 54, no. 11, pp. 1195-1206, 2012.

[56] F. Sambataro, V. P. Murty, J. H. Callicott et al., "Age-related alterations in default mode network: impact on working memory performance," Neurobiology of Aging, vol. 31, no. 5, pp. 839-852, 2010.

[57] M. Weiler, C. V. I. L. Teixeira, M. H. E. Nogueira et al., "Differences and the relationship in default mode network intrinsic activity and functional connectivity in mild Alzheimer's disease and amnestic mild cognitive impairment," Brain Connectivity, vol. 4, no. 8, pp. 567-574, 2014.

[58] R. Leech and D. J. Sharp, "The role of the posterior cingulate cortex in cognition and disease," Brain, vol. 137, no. 1, pp. 12-32, 2014.

[59] Z. Qi, X. Wu, Z. Wang et al., "Impairment and compensation coexist in amnestic MCI default mode network," NeuroImage, vol. 50, no. 1, pp. 48-55, 2010.

[60] S. J. Broyd, C. Demanuele, S. Debener, S. K. Helps, C. J. James, and E. J. S. Sonuga-Barke, "Default-mode brain dysfunction in mental disorders: a systematic review," Neuroscience \& Biobehavioral Reviews, vol. 33, no. 3, pp. 279-296, 2009.

[61] J. C. McVay, M. E. Meier, D. R. Touron, and M. J. Kane, "Aging ebbs the flow of thought: Adult age differences in mind wandering, executive control, and self-evaluation," Acta Psychologica, vol. 142, no. 1, pp. 136-147, 2013.

[62] J. S. Damoiseaux, C. F. Beckmann, E. J. S. Arigita et al., "Reduced resting-state brain activity in the 'default network' in normal aging," Cerebral Cortex, vol. 18, no. 8, pp. 1856-1864, 2008.

[63] S. Whitfield-Gabrieli and J. M. Ford, "Default mode network activity and connectivity in psychopathology," Annual Review of Clinical Psychology, vol. 8, pp. 49-76, 2012.

[64] C. O’Callaghan, J. M. Shine, S. J. G. Lewis, J. R. Andrews-Hanna, and M. Irish, "Shaped by our thoughts - A new task to assess spontaneous cognition and its associated neural correlates in the default network," Brain and Cognition, vol. 93, pp. 1-10, 2015.

[65] J. R. Andrews-Hanna, J. S. Reidler, J. Sepulcre, R. Poulin, and R. L. Buckner, "Functional-anatomic fractionation of the brain's default network," Neuron, vol. 65, no. 4, pp. 550-562, 2010. 
[66] A. M. C. Kelly, A. Di Martino, L. Q. Uddin et al., "Development of anterior cingulate functional connectivity from late childhood to early adulthood," Cerebral Cortex, vol. 19, no. 3, pp. 640657, 2009 .

[67] W. Gao, J. H. Gilmore, D. Shen, J. K. Smith, H. Zhu, and W. Lin, "The synchronization within and interaction between the default and dorsal attention networks in early infancy," Cerebral Cortex, vol. 23, no. 3, pp. 594-603, 2013.

[68] J. R. Andrews-Hanna, A. Z. Snyder, J. L. Vincent et al., "Disruption of large-scale brain systems in advanced aging," Neuron, vol. 56, no. 5, pp. 924-935, 2007.

[69] J.-T. Wu, H.-Z. Wu, C.-G. Yan et al., "Aging-related changes in the default mode network and its anti-correlated networks: a resting-state fMRI study," Neuroscience Letters, vol. 504, no. 1, pp. 62-67, 2011.

[70] C. Thai, Y. Y. Lim, V. L. Villemagne et al., "Amyloid-Related Memory Decline in Preclinical Alzheimer's Disease Is Dependent on APOE $\varepsilon 4$ and Is Detectable over 18-Months," PLoS ONE, vol. 10, no. 10, p. e0139082, 2015.

[71] A. P. Schultz, J. P. Chhatwal, T. Hedden et al., "Phases of Hyperconnectivity and Hypoconnectivity in the Default Mode and Salience Networks Track with Amyloid and Tau in Clinically Normal Individuals," The Journal of Neuroscience, vol. 37, no. 16, pp. 4323-4331, 2017.

[72] I. J. Bohr, E. Kenny, A. Blamire et al., "Resting-state functional connectivity in late-life depression: Higher global connectivity and more long distance connections," Frontiers in Psychiatry, vol. 3, Article ID Article 116, 2013.

[73] E. R. Kenny, J. T. O’Brien, D. A. Cousins et al., "Functional connectivity in late-life depression using resting-state functional magnetic resonance imaging," The American Journal of Geriatric Psychiatry, vol. 18, no. 7, pp. 643-651, 2010.

[74] C. E. Sexton, C. L. Allan, M. Le Masurier et al., "Magnetic resonance imaging in late-life depression: Multimodal examination of network disruption," Archives of General Psychiatry, vol. 69, no. 7, pp. 680-689, 2012.

[75] Z. Mirza, M. A. Kamal, A. M. Buzenadah, M. H. Al-Qahtani, and S. Karim, "Establishing genomic/transcriptomic links between Alzheimer's disease and type 2 diabetes mellitus by meta-analysis approach," CNS and Neurological Disorders Drug Targets, vol. 13, no. 3, pp. 501-516, 2014.

[76] Y. I. Sheline, D. M. Barcha, and J. L. Price, "The default mode network and self-referential processes in depression," Proceedings of the National Acadamy of Sciences of the United States of America, vol. 106, no. 6, pp. 1942-1947, 2009.

[77] S. B. Eickhoff, A. R. Laird, P. T. Fox, D. Bzdok, and L. Hensel, "Functional Segregation of the Human Dorsomedial Prefrontal Cortex," Cerebral Cortex, vol. 26, no. 1, pp. 304-321, 2016.

[78] D. Bzdok, R. Langner, L. Schilbach et al., "Segregation of the human medial prefrontal cortex in social cognition," Frontiers in Human Neuroscience, vol. 7, 2013.

[79] D. C. Steffens, L. Wang, K. J. Manning, and G. D. Pearlson, "Negative Affectivity, Aging, and Depression: Results From the Neurobiology of Late-Life Depression (NBOLD) Study," The American Journal of Geriatric Psychiatry, vol. 25, no. 10, pp. 11351149, 2017.

[80] M. Aghajani, I. M. Veer, M.-J. Van Tol et al., "Neuroticism and extraversion are associated with amygdala resting-state functional connectivity," Cognitive, Affective \& Behavioral Neuroscience, vol. 14, no. 2, pp. 836-848, 2014.

[81] M. G. Berman, S. Peltier, D. E. Nee, E. Kross, P. J. Deldin, and J. Jonides, "Depression, rumination and the default network,"
Social Cognitive and Affective Neuroscience, vol. 6, no. 5, pp. 548-555, 2011.

[82] P. C. Mulders, P. F. van Eijndhoven, A. H. Schene, C. F. Beckmann, and I. Tendolkar, "Resting-state functional connectivity in major depressive disorder: a review," Neuroscience \& Biobehavioral Reviews, vol. 56, pp. 330-344, 2015.

[83] J. A. Avery, W. C. Drevets, S. E. Moseman, J. Bodurka, J. C. Barcalow, and W. K. Simmons, "Major depressive disorder is associated with abnormal interoceptive activity and functional connectivity in the insula," Biological Psychiatry, vol. 76, no. 3, pp. 258-266, 2014.

[84] R. Kerestes, C. G. Davey, K. Stephanou, S. Whittle, and B. J. Harrison, "Functional brain imaging studies of youth depression: A systematic review," NeuroImage: Clinical, vol. 4, pp. 209-231, 2014.

[85] B. Sundermann, M. L. Beverborg, and B. Pfleiderer, "Toward literature-based feature selection for diagnostic classification: a meta-analysis of resting-state fMRI in depression," Frontiers in Human Neuroscience, vol. 8, article no. 692, 2014.

[86] C.-H. Liu, X. Ma, X. Wu et al., "Resting-state abnormal baseline brain activity in unipolar and bipolar depression," Neuroscience Letters, vol. 516, no. 2, pp. 202-206, 2012.

[87] M. S. Korgaonkar, N. J. Cooper, L. M. Williams, and S. M. Grieve, "Mapping inter-regional connectivity of the entire cortex to characterize major depressive disorder: a whole-brain diffusion tensor imaging tractography study," NeuroReport, vol. 23, no. 9, pp. 566-571, 2012.

[88] A. M. Schedlbauer, M. S. Copara, A. J. Watrous, and A. D. Ekstrom, "Multiple interacting brain areas underlie successful spatiotemporal memory retrieval in humans," Scientific Reports, vol. 4, article no. 6431, 2014.

[89] L. Schilbach, F. Hoffstaedter, V. Müller et al., “Transdiagnostic commonalities and differences in resting state functional connectivity of the default mode network in schizophrenia and major depression," NeuroImage: Clinical, vol. 10, pp. 326-335, 2016.

[90] J. V. Pardo, P. T. Fox, and M. E. Raichle, "Localization of a human system for sustained attention by positron emission tomography," Nature, vol. 349, no. 6304, pp. 61-64, 1991.

[91] M. I. Posner, J. A. Walker, F. J. Friedrich, and R. D. Rafal, "Effects of parietal injury on covert orienting of attention," The Journal of Neuroscience, vol. 4, no. 7, pp. 1863-1874, 1984.

[92] D. M. Wolpert, R. C. Miall, and M. Kawato, "Internal models in the cerebellum," Trends in Cognitive Sciences, vol. 2, no. 9, pp. 338-347, 1998.

[93] P. Johns, "Clinical Neuroscience," in International Standard Book Number: 978-0-443-10321-6, 2014.

[94] F. Barkhof, S. Haller, and S. A. R. B. Rombouts, "Resting-state functional MR imaging: A new window to the brain," Radiology, vol. 272, no. 1, pp. 29-49, 2014. 


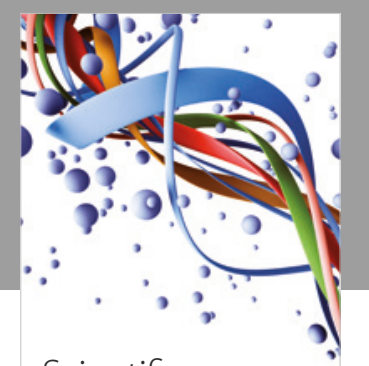

Scientifica
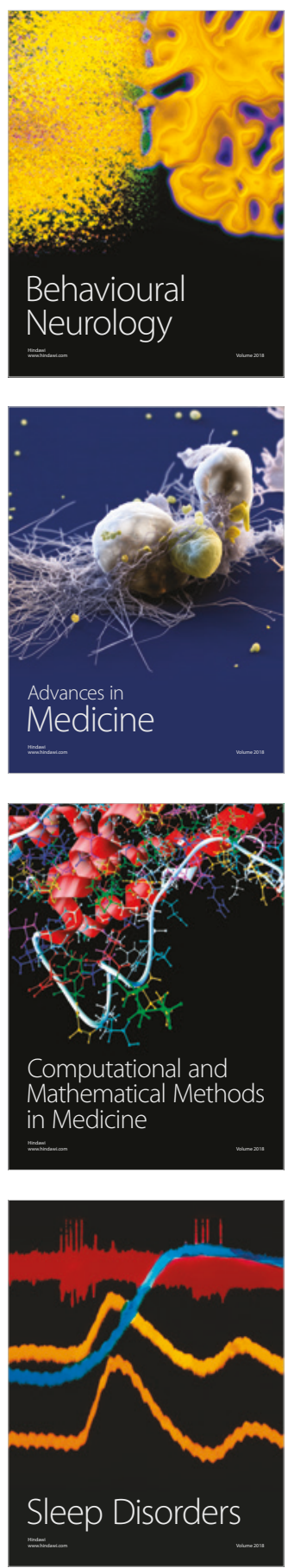

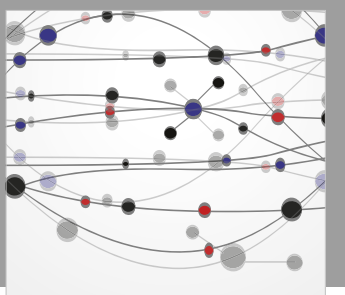

The Scientific World Journal

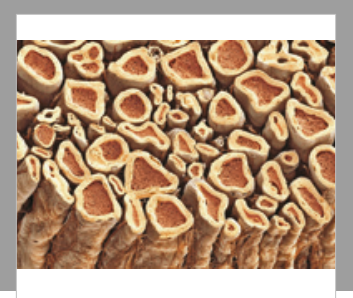

Case Reports in

Neurological Medicine

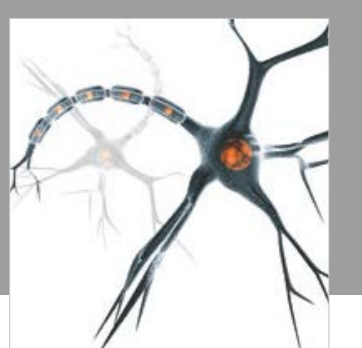

Neural Plasticity

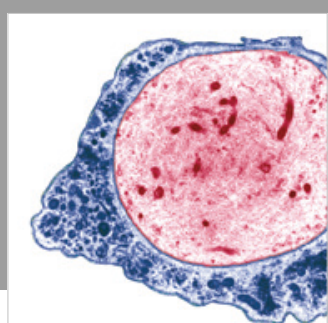

Multiple Sclerosis

International

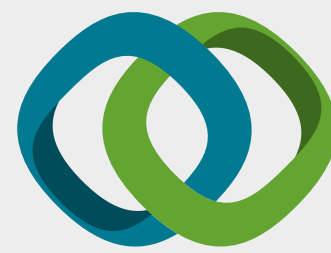

Hindawi

Submit your manuscripts at

www.hindawi.com
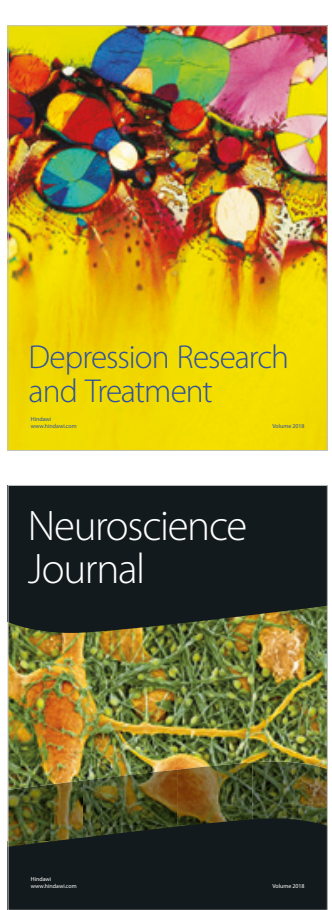

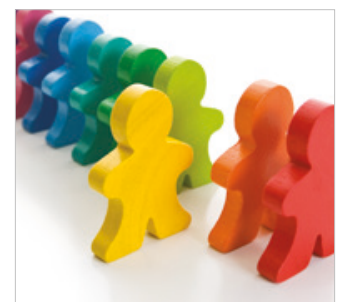

Autism

Research and Treatment
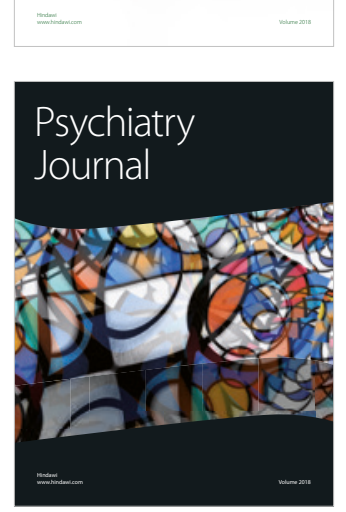
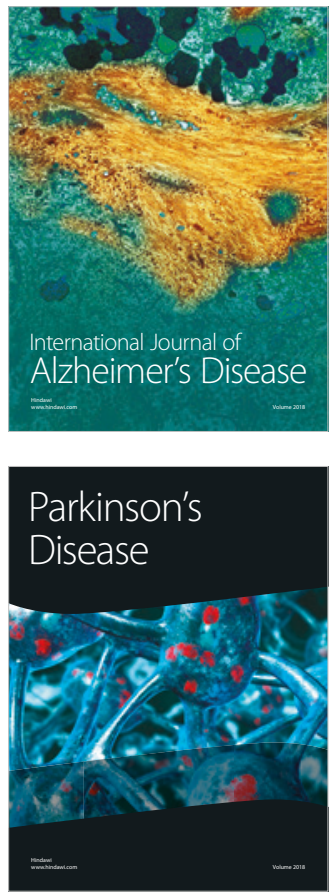
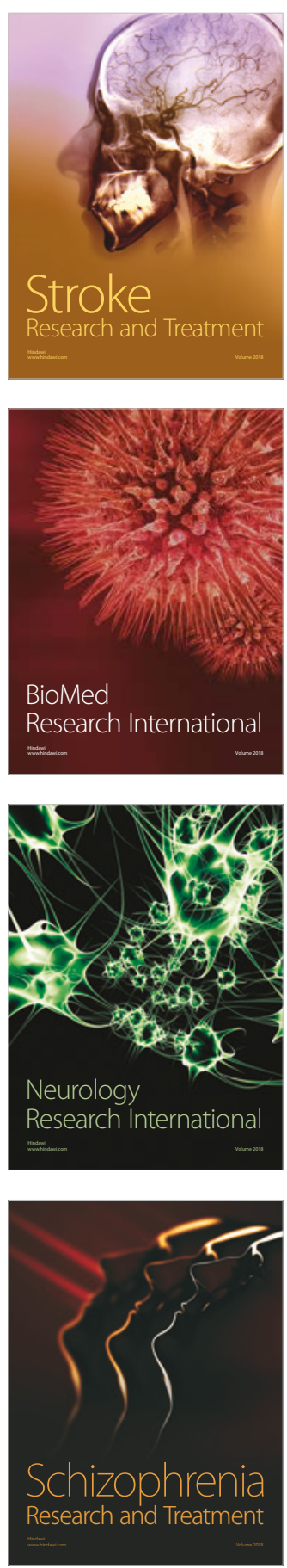